\title{
A Discrete Event System Specification (DEVS)-Based Model of Consanguinity
}

\author{
Noreen Akhtar, Muaz Niazi ${ }^{1,2}$, Farah Mustafa $^{1}$ and Amir Hussain ${ }^{2}$ \\ ${ }^{1}$ Department of Biosciences, \\ COMSATS Institute of IT, \\ Islamabad, Pakistan \\ ${ }^{2}$ Department of Computing Science and Mathematics, \\ University of Stirling, \\ Scotland, UK
}

\begin{abstract}
Consanguinity or inter-cousin marriage is a phenomenon quite prevalent in certain regions around the globe. Consanguineous parents have a higher risk of having offspring with congenital disorders. It is difficult to model large scale consanguineous parental populations because of disparate cultural issues unique to regions and cultures across the globe. Although consanguinity has previously been studied as a social problem, it has not been modeled from a biological perspective. Discrete Event System Specification (DEVS) is a powerful modeling formalism for the study of intricate details of real-world complex systems. In this paper, we have developed a DEVS model to get an insight into the role of consanguineous marriages in the evolution of congenital disorders in a population. As proof-of-concept, we further developed a consanguinity simulation model in Simio simulation software. Simulation results validated using population growth data show the effectiveness of this approach in the modeling of consanguinity in populations.

Copyright Notice: This is the author's version of the paper. Please cite the published paper as following1:

Noreen Akhtar, Muaz Niazi, Farah Mustafa, Amir Hussain, A discrete event system specification (DEVS)-based model of consanguinity, Journal of Theoretical Biology, Volume 285, Issue 1, 21 September 2011, Pages 103-112, ISSN 0022-5193, DOI: 10.1016/j.jtbi.2011.05.038. (http://www.sciencedirect.com/science/article/pii/S0022519311003031 ) Keywords: Consanguineous marriages; Simulation; Congenital disorder; Complex systems; DEVS formalism
\end{abstract}

${ }^{1}$ Copyright details: http://www.elsevier.com/wps/find/authorsview.authors/copyright 


\section{Introduction}

A consanguineous or inter-cousin marriage is a cultural tradition in many societies around the world [1]. A consanguineous marriage is formally defined as a marriage which is "solemnized among persons descending from the same stock or common ancestor with close biological relations" [2]. Although consanguinity may have some positive effects such as increase in specific measures of population fitness [3], primarily, it has been known to lead to an increased rate of birth defects, manifesting as severe recessive disorders [4-7]. Moreover, various studies have pointed out that consanguinity can lower many population fitness factors [8-10]. Despite this, inter-cousin marriages are prevailing and in fact spreading because of their socioeconomic usefulness amongst diverse populations.

Outside its social and cultural context, consanguinity from a biological perspective has not been modeled in the past. Computational modeling and simulation techniques have previously proven useful in developing insights and understanding of the dynamics of complex biological systems $[11,12]$. Large-scale consanguineous parental population is in essence, a possible domain for the application of simulation. Socially consanguineous population patterns tend to emerge within large segments of geographic areas despite cultural, linguistic and religious heterogeneity $[13,14]$, thereby making consanguinity modeling a challenging problem. Discrete Event System Specification (DEVS) formalism [15], a formal modeling and simulation framework, has previously been successfully exploited as a framework for modeling complex computer systems and, at times, certain natural and biological systems [16, 
17]. However, to the best of our knowledge, DEVS has not been previously used to model consanguinity.

The goal of the present study was to examine the role of consanguineous marriages in causing congenital defects using a modeling and simulation approach. With a dearth of modeling and simulation studies in the domain of consanguinity, there is a need to develop a fundamental formalism for modeling the complex system associated with congenital defect spread due to consanguinity. In this paper, we develop a DEVS framework for the formal modeling of consanguinity. As proof-of-concept, we demonstrate conversion of the formal model into an actual simulation model using Simio simulation software [18]. Validation of this model using real-world data shows that DEVS can be used effectively to model biological problems and Simio software can be useful for modeling complex biological and social problems.

\section{Background}

Consanguinity has been practiced in many cultures to secure certain family benefits such as dissemination of wealth among the same family, stability and better future [19, 20]. However, it has also been well demonstrated that consanguineous unions result in an increased rate of genetic defects in their offsprings [21, 22], including diabetes mellitus, cancers such as that of the cervix and the brain, and coronary artery disease $[7,10,23,24]$. Consanguinity has even been considered to contribute to an increase in incidents of hypertension [25, 26]. These negative health effects of consanguinity are due to single or multiple recessive genes expressed 
in the offspring [2]. Therefore, medically it is suggested that consanguineous marriages should be discouraged to avoid social damages.

The degree of relationship involved in consanguineous marriages affects the rate of birth defects proportionally [13]. Three relationship degrees are considered to have deleterious effects on human health that include first, second and third degree genetic associations, as shown in Figure 1 [27]. The first degree includes a person's parents and children. Siblings, grandparents, and grandchildren come under second degree of genetic relatedness, while aunts/uncles, nephews/nieces, great grandparents and great grandchildren come under the third degree (Figure 1). First-cousin marriages constitutes higher rate of consanguineous union and share twice the consanguinity (four times the degree of consanguinity of second cousins) as any other degree relationship, and are used as prototypical examples in studies of consanguinity [13]. First cousins share $50 \%$ genome, similar to their parents. That is why first cousin marriages are more discouraged than second or third degree unions. [28].

Due to population interactions complexities regarding consanguinity and large number of components involved, it is difficult to effectively study the behavior of consanguineous population along with congenital disorders. Many statistical studies have been conducted to study these interactions, but these studies have provided limited information regarding the resultant effects of consanguinity on a given population $[14,29,30]$. A few studies regarding simulation of consanguinity networks exist [31,32], but they have treated consanguinity as a social problem rather than a biological one. Since modeling and computer simulation techniques have previously proven useful for developing an understanding of the dynamics of complex biological systems [33, 34]; therefore this approach has been used to examine consanguinity in this study. 


\subsection{Modeling and Simulation}

Modeling is the process of producing models for a simulation study [35]. The main focus of modeling is on the input and output signal relation instead of detailed dynamics within the system [36]. Simulation is a tool which is used to simulate an abstract model or generate behavior of a particular system. Simulations are implemented with the help of a simulator software. If a model is a set of mathematical instructions, then simulator is a software which is used to execute these instructions and generate the behavior of the system of interest (Figure 2) [37]. The framework of modeling and simulation consists of four main entities [37]:

- Experimental frame

- $\quad$ Real/virtual source system to be simulated

- $\quad$ The model

- The simulator

The experimental frame specifies the environment under which the system is tested, while the source system is the environment (either real or virtual) to be modeled from which the data is gathered through observation.. The model is a mathematical representation of a system or structure to represent the real world behavior. The simulator is that computational system/software which obeys instructions of the model and generates behavior shown in Figure $2[37]$.

The modeling and simulation entities become significant only when they are properly related to each other. "Modeling Relation" shows the relationship between the behavior generated by the model, and how much it agrees with observed system behavior. On the other 
hand, "Simulation Relation" determines whether the simulator executes the model behavior correctly or not [37]. The framework of modeling includes many system specification formalisms, such as discrete time system specification (DTSS), differential equation system specification (DSS), and discrete event system specification (DEVS). These formalisms help to model systems in the most appropriate and effective manner during early development at the requirements and specification levels [37].

Modeling and computer simulation techniques have previously proven useful for understanding the dynamics of complex systems such as in ad-hoc and P2P networks [38], wireless sensor networks [39], detection of emergent behavior [40], breast cancer research [41, 42] and viral infections [43], etc. Recently, DEVS formalism has been used as a specification framework to model natural or biological systems effectively, such as interactions in nerve cells and geographic distribution of adult Mediterranean fruit fly [17, 44]. Therefore, in this study, we used the DEVS formalism to model potential effects of consanguinity in causing congenital defects.

\subsection{Discrete Event System Specification}

DEVS is a formal mathematical framework which is used to design models for discrete event simulation [37]. DEVS models are usually described as either atomic or coupled models which are defined as tuples:

\section{$(\mathrm{X}, \mathrm{Y}, \mathrm{S}, \boldsymbol{\delta e x t}, \boldsymbol{\delta i n t}, \lambda, \mathrm{ta})$ \\ [Atomic model] \\ $(\mathrm{X}, \mathrm{Y}, \mathrm{D},\{\mathrm{Mi}\},\{\mathrm{Ii}\},\{\mathrm{Zi}, \mathbf{j}\}$, select $)$ \\ [Coupled model]}

Behavior specification of a component within a system is defined by the Atomic DEVS model. Component's behavior specification is based on the time advance function ta(s), which 
keeps on changing after certain time period; therefore, it changes the initial state, s, of the component's behavior. During this constant time period, if any external event occurs and is received by the component as input, then a function, termed as "external transition", is implemented for the specific identification of the model's new transitioned state. Alternatively, if no external input enters in the component state and ta(s) defined time also passes by, then another function namely $\lambda$ is called out which displays the output. After the display of output of the current state, the transition function is again executed to find the possible next component state of the generated model [37].

DEVS's coupled model is based on several small components (models), termed Mi. These small models are connected to each other in a way that the output of one model acts as an input of some other model module $\{\mathrm{Ii}\}$. A defined function, $\mathrm{Zij}$, reads and understand the output from a component $i$ and then makes it compatible for another component $j$ as an input. This is termed as the coupling mechanism of the DEVS coupled model. The transition functions of the embedded models actively participate in changing the state of events; therefore a tiebreaking selector is used which helps in maintaining the order so that only one transition function remains on, and when the coupling is over, then the other transition function is prompted. Thus, a general statement can be made that under the coupled DEVS model, several atomic DEVS models can be embedded [37].

The major strategy behind DEVS is that the model and simulator work separately and the simulator does not depend on the model in a sense that it can run simulations regardless of what DEVS model represents [45]. DEVS formalisms are developed to improve system reliability, design time and comprehensibility [46]. Therefore, DEVS formalism provides a good framework to model consanguinity as a risk factor for many congenital disorders because 
it supports hierarchical and modular coupling construction for discrete events [47]. The impact of consanguinity as a genetic risk factor has not previously been modeled using formal methods. Therefore, our aim was to provide a DEVS framework to model consanguinity followed by conversion of DEVS model in simulation using Simio software and its validation.

\subsection{DEVS in Relation to Other Approaches:}

For the specification of model frameworks, various formalisms have been in use for decades. For example, Petri Nets [48], Discrete Time Specification [37], Finite State Automata [49] and Queuing Networks [50] have been widely used to specify system properties. In case of Discrete Time Specification (DTSS) formalism, inconsistency in the system state specifications is the major drawback because it treats time variable as a constant number, whereas in contrast, a real world system continuously changes with time. DEVS formal model specification framework, which uses mathematical notations to specify a system's behavioral characteristics, overcomes this problem by mainly focusing on the time " $t$ " variable, whose value continuously changes like other variables in a system. It does so by separating the system states and constant states by using transition functions which calculate constant states from current system states. DEVS maintains separation between the simulators and models to handle system state and constant state complexity.

For the modeling of complex biological systems, various statistical and computational methods are in use, but the development of complex models that can be used to simulate data is not always intuitive. There are certain reasons why we used DEVS approach to model consanguinity instead of using statistical and other computational approaches which are elaborated below: 


\subsubsection{DEVS vs. Statistical Distributions}

We used DEVS formalism to model consanguinity system. It is well known that statistical distributions cannot handle heterogeneities or micro behaviors of a system whose behavior and structure is not completely understood [51]. DEVS applies mathematical modeling for handling heterogeneous data, as demonstrated by Zeigler et al. (2000). They reasoned that DEVS data is represented in the form of a model in which one can observe the system state at any point and at any given time [37]. This is not the case with statistical tests since the intermediate values obtained through them do not provide any significant information as they are based on certain formulas [52]. Hence, whole test statistical distributions provide results in the form of a concrete estimated value or percentage, and although they have proved effective, they are too slow for real-time use. Using DEVS, ongoing experiments can be viewed theoretically at any step of the integrated subsystems [53], while in case of statistical tests, it is difficult to estimate the value of states that are continuously changing with respect to time [54]. Beside these reasons, many algebraic notations do not include the time element that DEVS inherits from its system theoretic origins.

\subsubsection{DEVS vs. Genetic Algorithms}

Genetic Algorithms (GA) are based on the theory of evolution [55]. The basis of this approach is that evolution always leads towards the survival of the fittest, but in case of consanguinity, it does not always go towards fitness and in fact has many harmful effects. Thus, even though in the case of malaria, it has been shown that consanguinity provides benefits by congregating malarial resistant genes in offspring [13], a slew of other studies have shown the harmful effects of consanguinity in the form of inheritance of abnormalities which 
pass from generation to generation $[7,10]$. Additionally, genetic algorithms have difficulty in handling two different state spaces at the same time, whereas DEVS framework can handle many scenarios of a system simultaneously by formally representing their structures [56].

It is also unreasonable to use genetic algorithms to study consanguinity because GA works by its own internal rules which cannot predict system behavior without testing them first on a simulation model [57]. On the other hand, DEVS framework can easily predict system behavior by changing its theoretical rules according to the particular system [53]. Furthermore, GA uses many methods for selecting fittest populations [58], but in consanguinity the focus is not on the selection of the best population; rather, the aim is to determine that certain threshold of consanguinity which could be hazardous for a population depending upon the phenotype being studied.

\section{Development of the DEVS Model of Consanguinity}

This section describes the DEVS model of consanguinity as a risk factor. As mentioned earlier, framework development of consanguinity system identifies key elements or entities and their relationships. Therefore, first we specified the basic entities of modeling and simulation of consanguinity, i.e., source/real system along with the experimental frame, model and simulator (Figures 2 and 3). The experimental frame includes region, religion, arranged marriages or self commitment, \% consanguinity, allelic frequency, consanguinity type (i.e., $1^{\text {st }}$

or $2^{\text {nd }}$ degree), and co-efficient of inbreeding. With consanguinity as the source system, the DEVS model and Simio simulator are shown in Figure 3. 
After specifying the entities, the DEVS formalism of consanguinity was built which is described below:

$\mathbf{M}=(\mathbf{X}, \mathbf{S}, \mathbf{Y}$, Sint, $\delta$ ext, $\lambda$, ta) where:

$\mathbf{M}$ is model representing the overall system

X: set of input values, i.e., region, religion, arrange/self commitment, \% consanguinity, allelic frequency, consanguinity type, and co-efficient of inbreeding

S: set of states, i.e., Birth event $\rightarrow$ Marriage event $\rightarrow$ Birth event $\rightarrow$ Marriage event. Between these two events, the probability of congenital disorders and deaths are also included

Y: set of output values, i.e., new populations with probability of congenital disorders due to consanguineous and non-consanguineous marriages

Sint: Internal transition function, i.e., if birth event occurs in consanguineous union, then there is a probability of congenital disorder risk and death events

Sext: External transition function, i.e., if male and female populations are available, then consanguineous marriage event will occur

$\lambda$ : Output Function, i.e., $S \rightarrow Y$

ta: Time advance function, i.e., new births, deaths and marriages per year

The model begins with a population growth submodel in order to understand how marriage and population growth events occurr, and then focuses on DEVS-based design of consanguinity to demonstrate its complexity. In the population growth model (Figure 4), initially two populations are used, i.e., male (MP) and female (FP) rather than the whole population to simplify model development and analysis. After separating both populations, marriage events occur followed by population growth. Under population growth, another source of offspring is added to generate children from resulting marriages randomly. This leads 
to a new population consisting of the number of total marriages and offspring. To simplify its presentation, we focused on the handling of interactions between male and female populations.

The population growth submodel design helped us to expand the model of consanguinity as a risk factor. Next, DEVS-based consanguinity formal model was developed with an embedded population growth submodel (Figure 5). In DEVS-based consanguinity formal model, initially the whole population (WP) is used and then separated into two groups, i.e., male (MP) and female (FP). This allows us to obtain male and female entities equally for marriage events. After combining both male and female populations, the group is split into two, i.e., consanguineous and non-consanguineous marriages. Consanguineous and nonconsanguineous marriages event now occur separately followed by population growth (births). At the end, we obtain a new population with the probability of consanguineous and nonconsanguineous marriage and their rate of offspring growth.

Next, we chose a simulation approach to implement the DEVS-based consanguinity model to maximize its utility.

\section{Simulation}

In the previous sections, we presented basic entities and framework of consanguinity using DEVS formalism. This section will further describe the implementation of consanguinity using the simulation software Simio. Simio makes simulation easier for decision making and enables users to solve more problems, more easily than ever before. It is based on the model object-oriented framework and facilitates building of 3D models [59]. In Simio, the basic concept of object-oriented framework is that classes define the behavior of objects [18]. Those classes, when placed together in a model, result in the emergence of system behavior from 
previously defined object interactions. Objects can be user-defined and can easily be added and extended in Simio. The basic object types in Simio are [59]:

- Fixed $\rightarrow$ a fixed location

- Source $\rightarrow$ generates entity objects

- Server $\rightarrow$ models a capacitated process

- Sink $\rightarrow$ destroys entities that have finished processing in the model

- Link $\rightarrow$ paths between objects

- Node $\rightarrow$ intersection between links

- Agent $\rightarrow$ unconstrained movement through free-space

- Entity $\rightarrow$ an agent that moves across links and enters objects

- Transporter $\rightarrow$ an entity that carries other entities

- Combiner $\rightarrow$ attaches the batched members to a parent entity

- Separator $\rightarrow$ separates batched members to a parent entity or makes copies of entity objects

\subsection{Simulation Model of Population Growth}

Model development in Simio begins with a population growth submodel, based on the flow diagram depicted in Figure 4. Many objects are used in this simulation, such as model entities (here entities mean individuals or parents), source, server, combiner, paths and sink which destroy entities. Two source objects are used to create the male and female populations to facilitate the correct manner of handling male and female entities. Then both of the source populations are combined in a 'Combiner' object that takes one of each entity, combines them (marriage), and sends them to Server1 named "Population Growth". Connecting the female- 
population source to the top entry point of the Combiner and connecting the male-population source to the bottom entry point of the combiner takes a parent and member. In this case, the default batch quantity of ' 1 ' is used since we require only one male and one female entity to attach.

To execute "Population Growth" event, a server is used within the "Add-On Process Triggers" section of "Properties", which adds a new logic named a 'Processed'. This directs the user to the "Processes" window with a new process called "Server1_Processed". Within this process, we can use a 'Create' step to create new entities (offspring) based on a distribution to determine the number of objects to create. When new entities are created, they are sent from the "Created Exit" of the "Create Step" and a "Transfer Step" step (Figure 6) can be used to transfer them from "free space" (where they are created) to a particular node - in this case, they can be transferred either to the Output@Server1, where they can exit with the parents, or they can be transferred into a "sink" where they can be counted.

In the Simio simulation, for generating offsprings, a "children" entity object (similar to MP and FP) are added that are animated in such a manner that one can see the difference between children and parents. This is not required, but may be desirable at times. To create offsprings, within the "Processes" window, use the "Create Step" to change the "Object Instance Name" to "Children" and "Number of Objects" to "Random.Discrete" distribution based on information provided regarding how many children per couple to create. For example, Figure 7 shows that $10 \%$ of the population has 0 children, $20 \%$ has 1 child, $30 \%$ has 2 children, $30 \%$ has 3 children, $8 \%$ has 4 children and $2 \%$ have 5.

To graphically depict children leaving Server1 (population growth) with the parent (instead of them all leaving simultaneously on top of each other), one can change the "Path 
Allow Passing" property to "False" so they are shown in a line (Figure 8). Data collected from simulations can provide information regarding the number of off springs calculated. The parents are never split, as a separator object is not used - but stay together with the animated parent entity.

The Population growth model generates male and female entities randomly and then these entities enter into a combiner object that triggers marriage events. The marriage events are followed by population growth (in which a random child population is created) and death event. After that this number is added into the new population (Figure 9).

\subsection{Simulation Model of Consanguinity}

This population growth submodel is further used in an expanded consanguinity model by splitting it into two submodels: consanguineous marriages and non-consanguineous marriages (Figure 10). In the same way as in the population growth model, the male (MP) and female (FP) population is used followed by the separation of this population into two groups based on consanguinity. The first group has male (MP_C) and female (FP_C) populations for consanguineous marriages and the second group holds male (MP_NC) and female (FP_NC) population for non-consanguineous marriages. Path weights are used to distribute these population entities accordingly. Then a combiner is used to tie male and female entities together in marriage. After reaching the queue of server named "Population Growth", birth events occur and generate offsprings randomly. The same process takes place with nonconsanguineous marriages with resulting birth events. The population is continuously updated based on marriages and generated offsprings (Figure 10). 
To differentiate offsprings which are generated randomly, an "Animation Property" is used which changes the appearance of the child entity. This dynamic property can be applied by using the "Additional Symbol" icon which generates multiple symbols of single entity until the desired number of symbols has been achieved. Two symbols of a single entity to represent male and female children were created in both population growth and consanguinity models (Figure 11). After creating multiple desired symbols, the "Model Entity Properties $>$ Animation $>$ Random Symbol Property" was set to True, to allow each new object to be randomly assigned a symbol from the "Additional Symbol List".

\section{Results and Discussion}

The consanguinity model thus developed was used to simulate real data for the validity of the model. Simulations were run at least 50 times and the results were exported into an Excel sheet with comprehensive data and statistics regarding the model behavior. Here, we will discuss results with the help of tables generated through Simio pivot grid or reports.

The first step towards testing our model was to validate the population growth model. This was done using data for Saudi Arabia available in the World Factbook [60]. Data from Saudi Arabia was chosen for the validation purposes since it is a society with high consanguinity rates for which a lot of data was available that we could use for the validation purposes.

\subsection{Population Growth Model Validation}

We validated our Population Growth Model by plotting the real data of Saudi population from the years 2005-2009 [60] side by side with results from simulation of the same data using our Population Growth Model. As can be seen, the predicted population came within 3\% of the 
actual population (Table 1 and Figure 12), precisely within the margin of error for actual Saudi Arabia population sampling that was $\pm 3 \%$ [60]. Furthermore, plotting of the data with time showed that the growth curves of the predicted and expected populations were the same, revealing an excellent correlation of the expected growth rate with the actual one (Figure 12).

Similarly, Simio was used to predict the number of male and female members of the Saudi population generated over the period from 2006-2009 using total population data, death rate, and male:female sex ratio from the year 2005 from the World Factbook [60]. As can be seen (Figures 13 and 14), the actual and predicted gender-specific ratio observed for the year 2009 was similar in which the male population remained higher than the female population, a trend observed worldwide [60]. However, in terms of absolute numbers, there was a $14 \%$ difference between the predicted and actual gender-specific population numbers for the year 2009 [61], which probably reflects inaccuracies of reporting the actual numbers in the 2009 census, and/or use of sex ratio figures from different studies (World Factbook data [60] verses World Population Prospects [61]).

Thus, test of our Population Growth Model revealed that overall its simulation reflected real world data trends, and can be used for further simulation of consanguinity in a population.

\subsection{Consanguinity Model Validation}

To validate the consanguinity model, we needed specific data that was not available in the literature. Therefore, we used data available from different studies to determine whether our model could give us results as close to real life as possible. Towards this end, gender and death rate data for Saudi Arabia (2006) was used from the World Factbook [60]. The sex distribution of Saudi Arabia was 1.33 male(s)/female between the ages of 15-64 years [60]. 
We selected this defined age range since it covers the reproductive time period of individuals which directly relates to consanguineous or non-consanguineous marriages. Our consanguinity model separates the male and female population into two groups (Figure 10). The first group has male (MP_C) and female (FP_C) population for consanguineous marriages. Here, the distribution rate for consanguineous union was taken as $30 \%$ based on average rates of marriages between first cousins among Saudi populations [62]. Conversely, for nonconsanguineous marriages, the paths weights were assigned as 70 (Figure 10). To correctly reflect the growth rate, a death rate of 2.58/1,000 for the year 2006 was taken from the World Factbook for Saudi Arabia and assigned to accurately predict the population growth [60]. After assigning these parameters, comprehensive data was generated by our consanguinity model, shown in Table 2.

Table 2 demonstrates the total number of consanguineous and non-consanguineous marriages along with the population growth as a result of these marriages for the year 2006 . Since the actual data of consanguineous and non-consanguineous population does not exist, in order to validate our model, we predicted the total population of Saudi Arabia for the year 2006 by adding both consanguineous and non-consanguineous populations obtained through the simulation analysis and compared it to the total population of Saudia Arabia estimated actually for the year 2006 (Table 2). The two numbers came within 3.2\%--the error rate of the data collection [60], revealing once again an excellent correlation of the predicted results with that of the actual data, though in an indirect manner.

\section{Conclusions and Future Work:}

In this study, we have developed a DEVS model of population growth and used it to study the in silico emergence of consanguinity in the offsprings. The main idea of this study 
was to take the first steps towards answering questions such as what are the rates of consanguinity which can impact the emergence of birth defects in a population. It is important to model consanguinity in order to acquire a complete picture of congenital defect trends. Our contribution is a DEVS-based model of consanguinity which can predict the level of consanguinity in a population followed by simulation using the Simio simulation software. Validation of our model using real-world data shows that DEVS can be used effectively to model biological problems. In future, we plan on applying the DEVS formalism to specific congenital disorders due to consanguinity to evaluate the probability of congenital defects due to consanguineous and non-consanguineous marriages.

\section{Acknowledgments}

The authors wish to acknowledge the use of Simio Simulation Software Services. This work was made possible by an academic grant from Simio LLC to CIIT and a research grant to FM by the Higher Education Commission (HEC), Pakistan. 


\section{References}

[1] S. Al-Awadi, et al., "Consanguinity among the Kuwaiti population," Clinical genetics, vol. 27, pp. 483-486, 1985.

[2] M. Mehndiratta, et al., "Arranged marriage, consanguinity and epilepsy," Neurology Asia, vol. 12, pp. 15-17, 2007.

[3] S. Denic, et al., "Consanguineous marriages and endemic malaria: can inbreeding increase population fitness?," Malaria Journal, vol. 7, p. 150, 2008.

[4] B. Modell and A. Darr, "Genetic counselling and customary consanguineous marriage," Nat Rev Genet, vol. 3, pp. 225-229, 2002.

[5] P. Strømme, et al., "Parental consanguinity is associated with a seven-fold increased risk of progressive encephalopathy: A cohort study from Oslo, Norway," European Journal of Paediatric Neurology, vol. 14, pp. 138-145, 2010.

[6] S. Becker, "Consanguinity and congenital heart disease in Saudi Arabia.," Am J Med Genet, vol. 13, pp. 8-13, 2001.

[7] I. Rudan, et al., "Inbreeding and risk of late onset complex disease," Journal of Medical Genetics, vol. 40, p. 925, 2003.

[8] A. Bittles, "Consanguinity and its relevance to clinical genetics," Clinical Genetics, vol. 60, pp. 89-98, 2001.

[9] S. Amudha, et al., "Consanguinity and chromosomal abnormality," Indian Journal of Human Genetics, vol. 11, pp. 108-110, 2005 
[10] A. Bener, et al., "Consanguineous marriages and their effects on common adult diseases: studies from an endogamous population," Medical Principles and Practice, vol. 16, p. 262, 2007.

[11] T. Meng, et al., "Modeling and simulation of biological systems with stochasticity," In silico biology, vol. 4, pp. 293-309, 2004.

[12] J. Cundiff and F. Agblevor, "Simulation of biological systems," Unpublished class notes. Biological Systems Engineering, Virginia Tech. Blacksburg, VA, vol. 24061, 1998.

[13] S. Denic and M. Nicholls, "Genetic benefits of consanguinity through selection of genotypes protective against malaria," Human Biology, vol. 79, pp. 145-158, 2007.

[14] Y. Imaizumi, "A recent survey of consanguineous marriages in Japan: religion and socioeconomic class effects," Annals of human biology, vol. 13, pp. 317-330, 1986.

[15] T. Kim, et al., "DEVS framework for systems development: unified specification for logical analysis, performance evaluation and implementation, Discrete event modelng and simulation technologies: a tapestry of systems and AI-based theories and methodologies," Discrete Event Modeling and Simulation Technologies: A Tapestry of Systems and Ai-Based Theories and Methodologies: A Tribute to the 60th Birthday of Bernard P. Zeigler, p. 131, 2001.

[16] Z. Wang, "Cell Biology Simulation Using Devs Combined with SBML " Master Of Science, Department of Electrical and Computer Engineering, The University of Arizona, 2009.

[17] R. Goldstein and G. Wainer, "DEVS-based design of spatial simulations of biological systems," Winter Simulation Conference (WSC), Proceedings of the 2009, , pp. 743-754, 2010. 
[18] C. Pegden, "SIMIO: a new simulation system based on intelligent objects," 2008, pp. 2293-2300.

[19] B. Modell, et al., "Community control of genetic and congenital disorders," WHO EMRO Technical Publication series, vol. 24, 1997.

[20] M. Khlat, Endogamy in the Arab world. In: Teebi AS, Farag TI (eds) Genetic disorders among Arab populations. New York: Oxford University Press, pp. 63-80, 1997.

[21] M. El Mouzan, et al., "Consanguinity and major genetic disorders in Saudi children: a community-based cross-sectional study," Annals of Saudi Medicine, vol. 28, p. 169, 2008.

[22] G. Tadmouri, et al., "Consanguinity and reproductive health among Arabs," Reproductive Health, vol. 6, p. 17, 2009.

[23] I. Rudan, "Campbell h, Carothers aD, hastie $\mathrm{nD}$, Wright $\mathrm{aF}, "$ Contribution of consanguinuity to polygenic and multifactorial diseases. nat Genet, vol. 38, pp. 1224-5, 2006.

[24] S. Denic, "Consanguinity as risk factor for cervical carcinoma," Medical Hypotheses, vol. 60, pp. 321-324, 2003.

[25] I. Rudan, et al., "Inbreeding and the genetic complexity of human hypertension," Genetics, vol. 163, p. 1011, 2003.

[26] B. Charlesworth and K. Hughes, "Age-specific inbreeding depression and components of genetic variance in relation to the evolution of senescence," Proceedings of the National Academy of Sciences of the United States of America, vol. 93, p. 6140, 1996.

[27] County, "Tell the brother-in-law to find work elsewhere," Taxes Association of Counties, vol. 11, 1999. 
[28] G. Carey, Human genetics for the social sciences: Sage Publications, Inc, 2003.

[29] M. Hader, et al., "Consanguinity and increased risk for schizophrenia in Egypt," Schizophrenia research, vol. 120, pp. 108-112, 2010.

[30] L. Jaber, et al., "High incidence of central nervous system malformations associated with marked parental consanguinity in an Israeli Arab community," Biomedicine \& Pharmacotherapy, vol. 48, pp. 351-354, 1994.

[31] D. White, "Controlled simulation of marriage systems," Journal of Artificial Societies and Social Simulation, vol. 2, p. 5, 1999.

[32] D. White, "Ring cohesion theory in marriage and social networks," Mathématiques et sciences humaines, vol. 168, pp. 5-28, 2004.

[33] G. Ermentrout and L. Edelstein-Keshet, "Cellular automata approaches to biological modeling," Journal of Theoretical Biology, vol. 160, pp. 97-97, 1993.

[34] L. Saiz and M. Klein, "Computer simulation studies of model biological membranes," Acc. Chem. Res, vol. 35, pp. 482-489, 2002.

[35] A. Maria, "Introduction to modelling and simulation," in Proceedings of the 1997 Winter Simulation Conference, pp. 7-13, 1997.

[36] A. Jauhiainen, "Evaluation and Development of Methods for Identification of Biochemical Networks," Master's, Department of Physics and Measurement Technology, Link“opings universitet, Sweden, 2005.

[37] B. Zeigler, et al., Theory of Modeling and Simulation., 2nd ed. San Diego, CA.: Academic Press, 2000. 
[38] M. Niazi and A. Hussain, " Agent based Tools for Modeling and Simulation of SelfOrganization in Peer-to-Peer, Ad-Hoc and other Complex Networks," IEEE Communications Magazine, vol. 47, pp. 163 - 173., March 2009.

[39] M. A. Niazi and A. Hussain, "A Novel Agent-Based Simulation Framework for Sensing in Complex Adaptive Environments," Sensors Journal, IEEE, vol. 11, pp. 404-412, 2011.

[40] M. Niazi and A. Hussain, "Sensing Emergence in Complex Systems," IEEE Sensors Journal, vol. In-press, 2011.

[41] A. Siddiqa, et al., "A new hybrid agent-based modeling \& simulation decision support system for breast cancer data analysis," in Information and Communication Technologies, 2009. ICICT '09. International Conference on, pp. 134-139, 2009.

[42] R. Mukhopadhyay, et al., "Promotion of variant human mammary epithelial cell outgrowth by ionizing radiation: an agent-based model supported by in vitro studies," Breast Cancer Res, vol. 12, p. R11, Feb 102010.

[43] J. Itakura, et al., "Reproducibility and usability of chronic virus infection model using agent-based simulation; comparing with a mathematical model," Biosystems, vol. 99, pp. 70-8, Jan 2010.

[44] F. Bernardi, et al., "A generic framework for environmental modeling and simulation,", In Proceedings of the 2003 IEEE International Conference on Systems, Man and Cybernetics, October 5-8, in Washington, DC., vol. 2, pp. 1810-1815, 2003.

[45] R. Goldstein and G. Wainer, "DEVS-based design of spatial simulations of biological systems," in Winter Simulation Conference (WSC), Proceedings of the 2009, , pp. 743$754,2010$. 
[46] A. I. Wasserman, "The evolution of specification techniques," ACM Computing Surveys (CSUR), pp. 134-137, 1977.

[47] H. Shang and G. Wainer, "A simulation algorithm for dynamic structure DEVS modeling," WSC '06 Proceedings of the 38th conference on Winter simulation, pp. 815$822,2006$.

[48] D. Kartson, et al., Modelling with generalized stochastic Petri nets: John Wiley \& Sons, Inc. New York, NY, USA, 1994.

[49] M. Mohri, "On some applications of finite-state automata theory to natural language processing," Natural Language Engineering, vol. 2, pp. 61-80, 1996.

[50] M. Reiser and H. Kobayashi, "Queuing networks with multiple closed chains: theory and computational algorithms," IBM Journal of Research and Development, vol. 19, pp. 283294, 1975.

[51] P. Dutilleul and P. Legendre, "Spatial Heterogeneity against Heteroscedasticity: An Ecological Paradigm versus a Statistical Concept," Oikos, vol. 66, pp. 152-171, 1993.

[52] V. Lepetit, et al., "Randomized trees for real-time keypoint recognition," In CVPR '05 Vol.2, pages 775-781, 2005.

[53] B. P. Zeigler and H. S. Sarjoughian, "DEVS Component-Based M\&S Framework: An Introduction," Proceedings of the 2002 AI, Simulation and Planning in High Autonomy Systems. Fernando J.Barros and Norbert Giambiasi Editors.Lisbon, Portugal, April 2002.

[54] W. Uther and M. Veloso, "Generalizing Adversarial Reinforcement Learning,", Technical Report, Carnegie Mellon University, Pittsburgh, PA, 1997. Unpublished., 1997. 
[55] M. Mitchell, An introduction to genetic algorithms: The MIT press, Cambridge, Massachusetts 1998.

[56] C. M. Close, Frederick, D. K., and Newell, J. C., Modeling and Analysis of Dynamic Systems. New York, NY USA: John Wiley \& Sons, 2002.

[57] G. Eoyang, "Genetic Algorithm as Decision Support Tool," Chaos Limited, 1996.

[58] M. F. Bramlette, "Initialization, mutation and selection methods in genetic algorithms for function optimization," San Diego, CA: Morgan Kaufmann. vol. 100, p.100-107, 1991.

[59] C. Pegden, "Introduction to Simio," Winter Simulation Conference, pp. 229-235, 2008.

[60] Central Intelligence Agency (CIA). Saudi Arabia. The World Factbook 2006. Available at: http://www.cia.gov/cia/publications/factbook/.

[61] UN Statistics Division, Department of Economic and Social Affairs. "World Population Prospects: $\quad$ The 2008 Revision". Available at: http://www.geohive.com/earth/pop_gender.aspx

[62] L. Al-Gazali, et al., "Genetic disorders in the Arab world," BMJ, vol. 333, p. 831, 2006. 
Table 1: Population Growth Statistics via Simio Simulations. The year column shows the period over which female and male population data was generated along with the growth of the total population.

\begin{tabular}{|c|c|c|c|}
\hline Year & $\begin{array}{c}\text { Actual Population } \\
\text { Growth of Saudi } \\
\text { Arabia* }\end{array}$ & $\begin{array}{c}\text { Predicted Population Growth } \\
\text { Rate of Saudi Arabia }\end{array}$ & $\begin{array}{c}\text { \% Difference Between } \\
\text { Actual and Predicted } \\
\text { Growth Rates }\end{array}$ \\
\hline $\mathbf{2 0 0 5}$ & 26417599 & 25655000 & 2.89 \\
\hline $\mathbf{2 0 0 6}$ & 27019731 & 26231000 & 2.92 \\
\hline $\mathbf{2 0 0 7}$ & 27601038 & 26790600 & 2.94 \\
\hline $\mathbf{2 0 0 8}$ & 28146656 & 27411500 & 2.46 \\
\hline $\mathbf{2 0 0 9}$ & 28686633 & 27980600 & \\
\hline
\end{tabular}

*Actual data available in CIA World Factbook [60]. 
Table 2: Statistics on total number of consanguinity model entities created using Simio simulation and comparison with real data.

\begin{tabular}{|c|c|c|c|}
\hline Objects Name & $\begin{array}{c}\text { Actual Statistics } \\
\text { of Saudi Arabia } \\
\text { from year 2006* }\end{array}$ & $\begin{array}{c}\text { Predicted Statistics } \\
\text { from Consanguinity } \\
\text { Model }\end{array}$ & \% Difference \\
\hline Consanguineous marriage(s) & - & 3154352 & \\
\hline Non-consanguineous marriage(s) & - & 9898900 & \\
\hline Consanguineous population growth & - & 9628005 & \\
\hline Non-consanguineous population growth & - & 18290500 & 3.22 \\
\hline Total population growth & 27019731 & 27918505 & \\
\hline
\end{tabular}

*Actual data available in CIA World Factbook [60]. 


\section{Figure Captions:}

Figure 1: Degree relationships between family members. Each circle represents the degree of consanguinity (genetic relatedness) with the person in the middle of the figure. (Figure modified from [27]).

Figure 2: The basic entities in modeling and simulation and their relationship to each other. Experimental frame specifies the conditions or environment in which system is experimented with, source system is the real or virtual system which is to be modeled, model is a mathematical representation of any system or structure, data is usually gathered by observing it, and simulator is a software which follows model instructions and generate particular behavior of a real system.

Figure 3: Basic entities of modeling and simulation of consanguinity. This figure shows the main entities of consanguinity model which specifies experimental frame (population, allelic frequency, region, religion, cousin and consanguinity type, etc.), source system (consanguinity), model (DEVS-based) and simulator (Simio simulation software) for DEVSbased model of consanguinity.

Figure 4: Flow diagram of population growth. This flow diagram shows population growth model which initialized with two populations, i.e., male (MP) and female (FP). After separating both populations, marriage events occur followed by population growth. Under population growth, another source of offspring (child) is added to generate children from resulting marriages randomly. This leads to a new population consisting of the number of total marriages and offspring.

Figure 5: DEVS-based consanguinity model. Flow diagram of DEVS-based consanguinity model. MP, male population; FP, female population; Children_C, children resulting from 
consanguineous union; Children_NC, children resulting from non-consanguineous union. This model initiates with male and female populations. After combining these two populations, the group is split into: consanguineous and non-consanguineous marriages. Consanguineous and non-consanguineous marriages event now occur separately followed by population growth (births) which include offspring source to generate children randomly. At the end, a new population with the probability of consanguineous and non-consanguineous populations with their rate of offspring growths are created.

Figure 6: Add-On Process used in the Population Growth Model to create offsprings randomly.

Figure 7: Snapshot of “Create Step” for random offspring generation. This figure shows the properties for creating offspring randomly. To create offsprings, within the "Processes" window, use the "Create Step" to change the "Object Instance Name" to "Child" and "Number of Objects" to "Random.Discrete" distribution based on information provided regarding how many children per couple to create. For example, this figure shows that $10 \%$ of the population has 0 children, $20 \%$ has 1 child, $30 \%$ has 2 children, $30 \%$ has 3 children, $8 \%$ has 4 children and $2 \%$ have 5 .

Figure 8: Parent and offspring move together into a new population object as discrete entities in a line. This figure shows the children leaving the Server1 (population growth) with the parent graphically and then counted in sink object (new population).

Figure 9: Two and 3D view of the Simio complete population growth submodel. DEVSbased population growth submodel in 3D, using Simio simulation software.

Figure 10: Model of consanguinity. MP_C, male population for consanguineous marriages; FP_C, female population for consanguineous marriages; Children_C, children resulted from consanguineous union; Marriage_C, consanguineous union; PopulationG_C, 
consanguineous population growth; NewPopulation_C, new consanguineous population; MP_NC, male population for non-consanguineous $\quad$ marriages; $\quad$ FP_NC, female population for non-consanguineous marriages; Children_NC, children resulted from nonconsanguineous union; Marriage_NC, non-consanguineous union; PopulationG_C, nonconsanguineous population growth; NewPopulation_C, new non-consanguineous population.

Figure 11: Adding additional symbols in Consanguinity Model. This property helps to create multiple desired symbols.

Figure 12: Population Growth Model validation with Saudi population data. Graph comparing the original Saudi population growth data from the years 2005-2009 with the simulation data generated via Simio in SPSS.

Figure 13: Male and female statistics from 2005-2009, generated using Simio simulation software generated using SPSS.

Figure 14: Gender-specific distribution of the Saudi population in 2009 predicted by means of Simio using population growth data from 2006 generated using SPSS. 


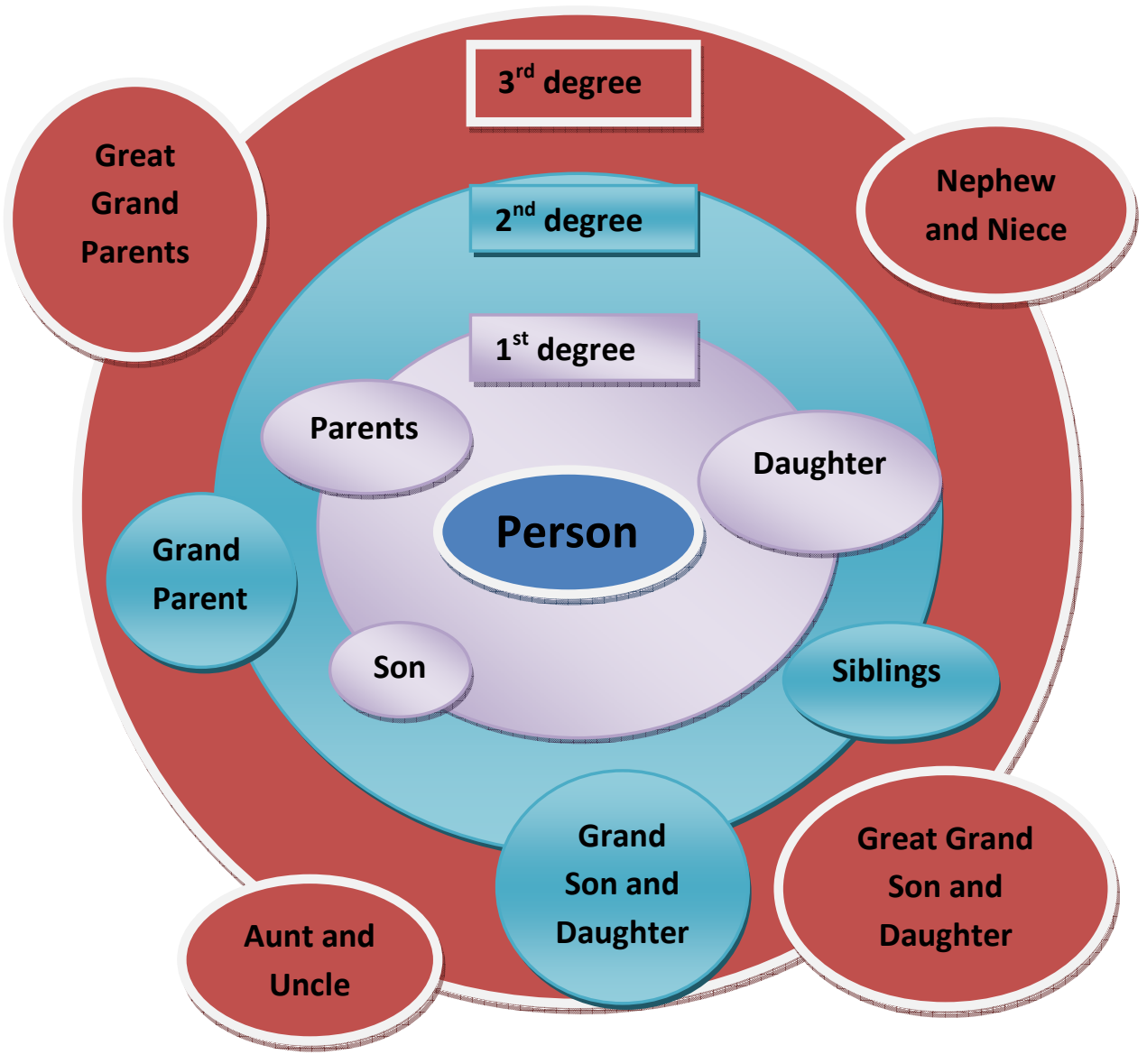

Figure 1. 


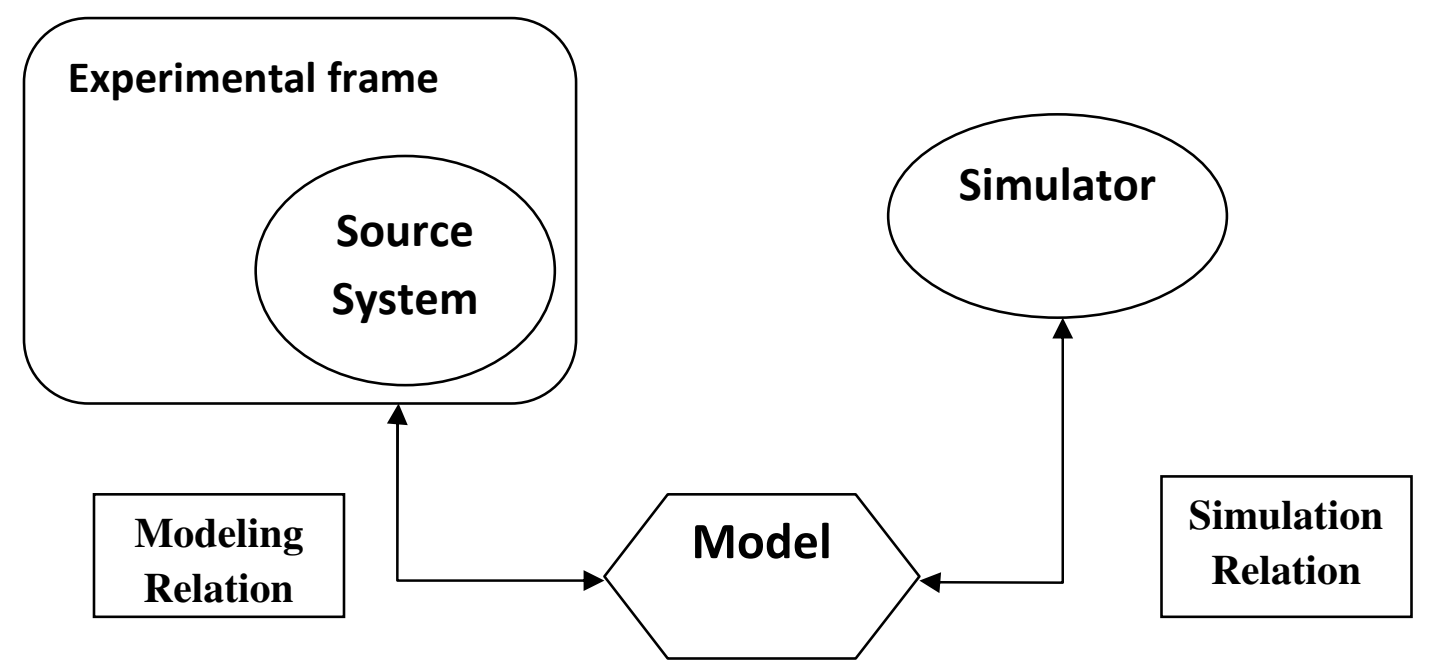

Figure 2. 


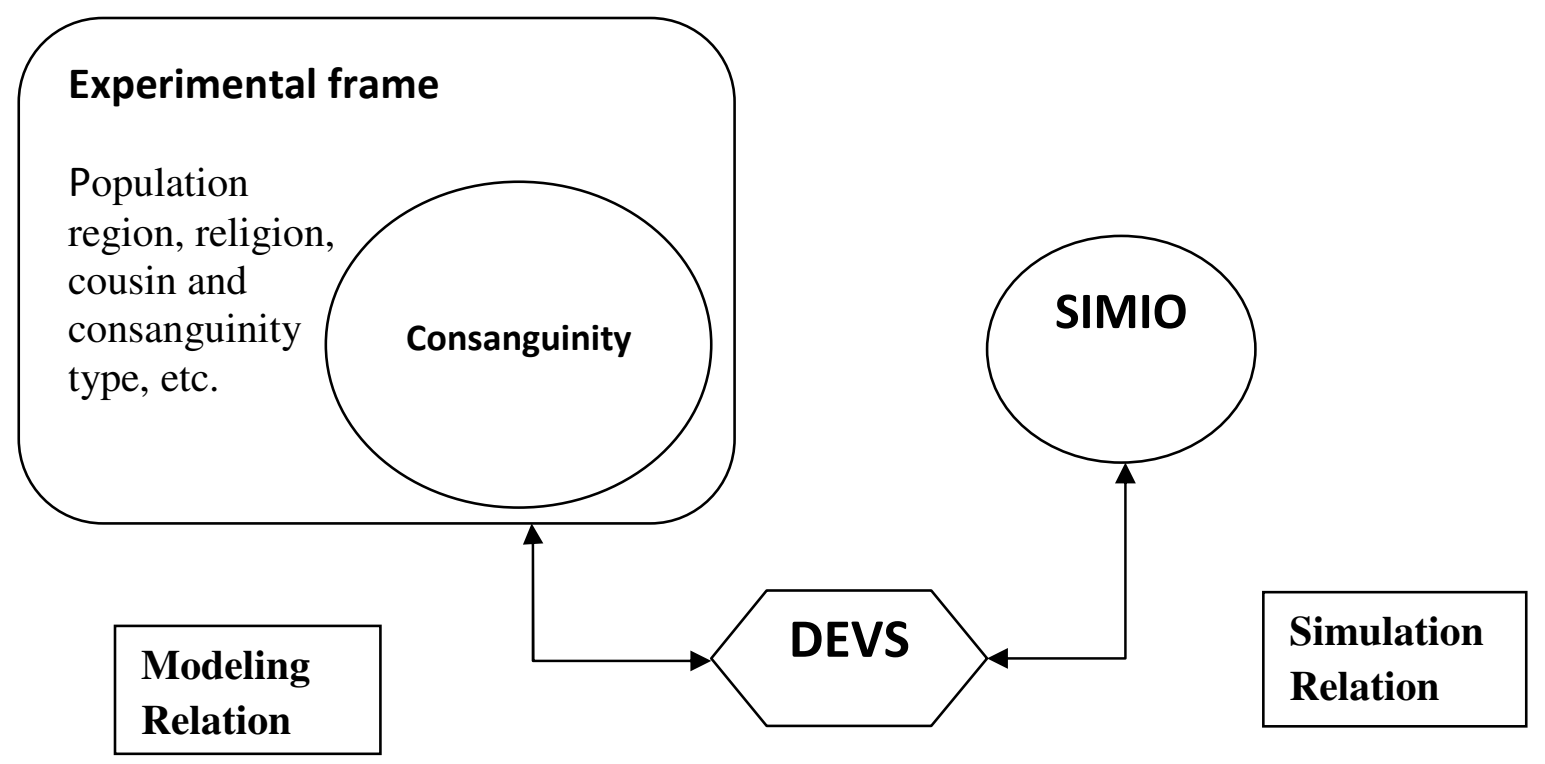

Figure 3. 


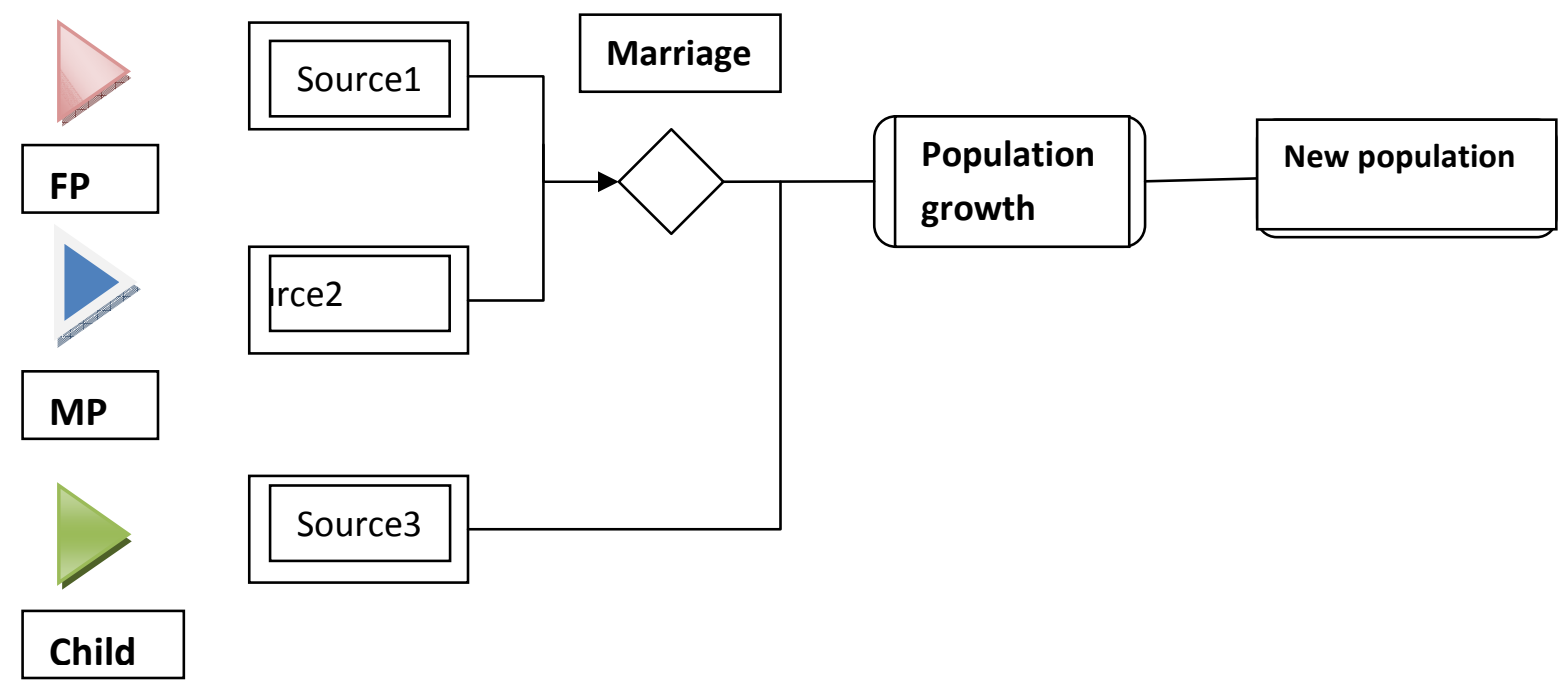

Figure 4. 


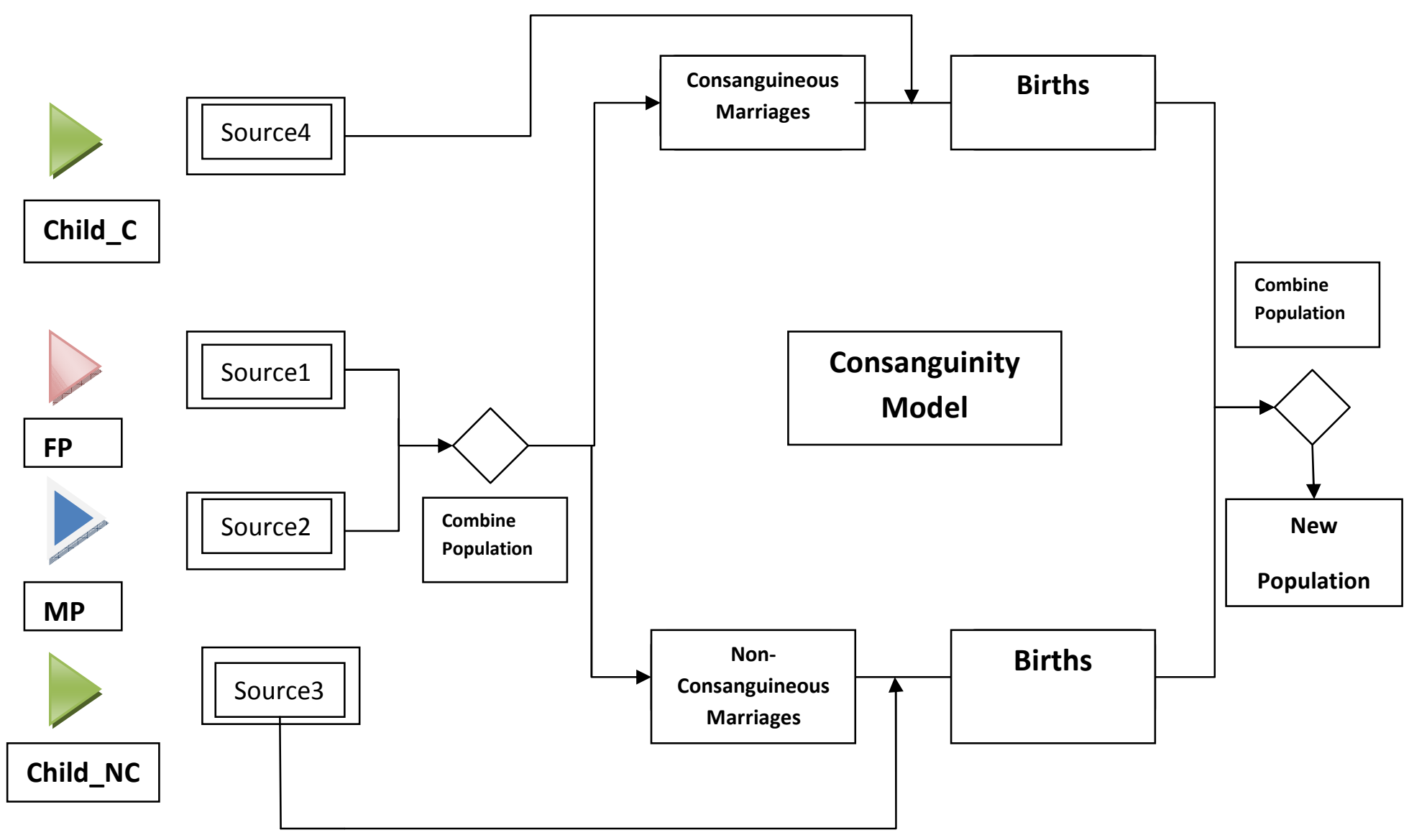

Figure 5. 
$\because$ PopulationGrowth AddOn Processes

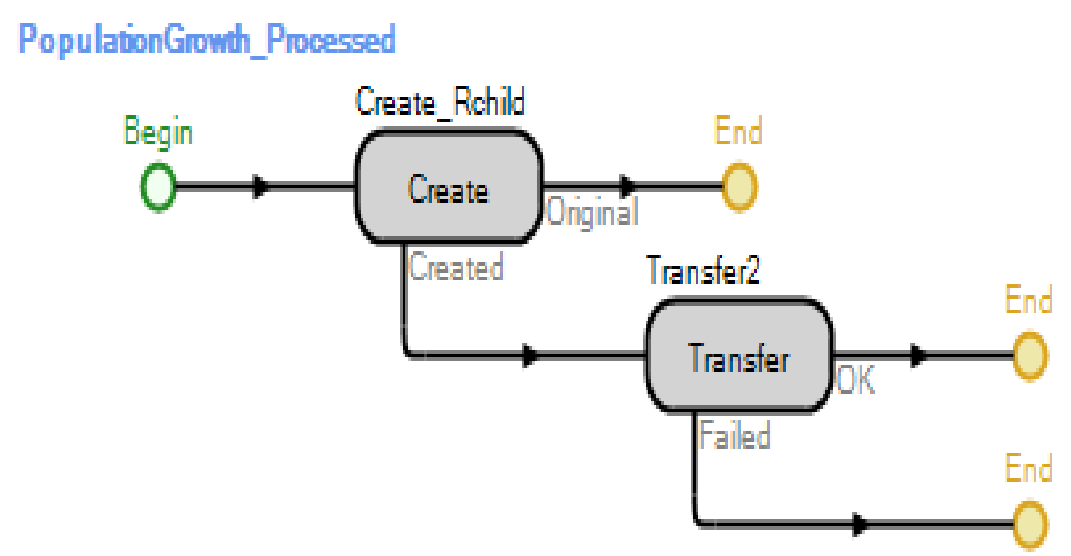

Figure 6. 


\begin{tabular}{|c|c|}
\hline \multicolumn{2}{|c|}{ Properties Crestel (Create step Instance) } \\
\hline \multicolumn{2}{|l|}{ E Basic Logic } \\
\hline Geste Type & Newobjet: \\
\hline Object Instance Name & Child \\
\hline Number of otjects & Random.Discrete $(0,1,1,3,2,6,6,3,90,4,96,5,1)$ \\
\hline \multicolumn{2}{|l|}{ \pm Advanced 0ptions } \\
\hline$\doteq$ General & \\
\hline
\end{tabular}

Figure 7. 


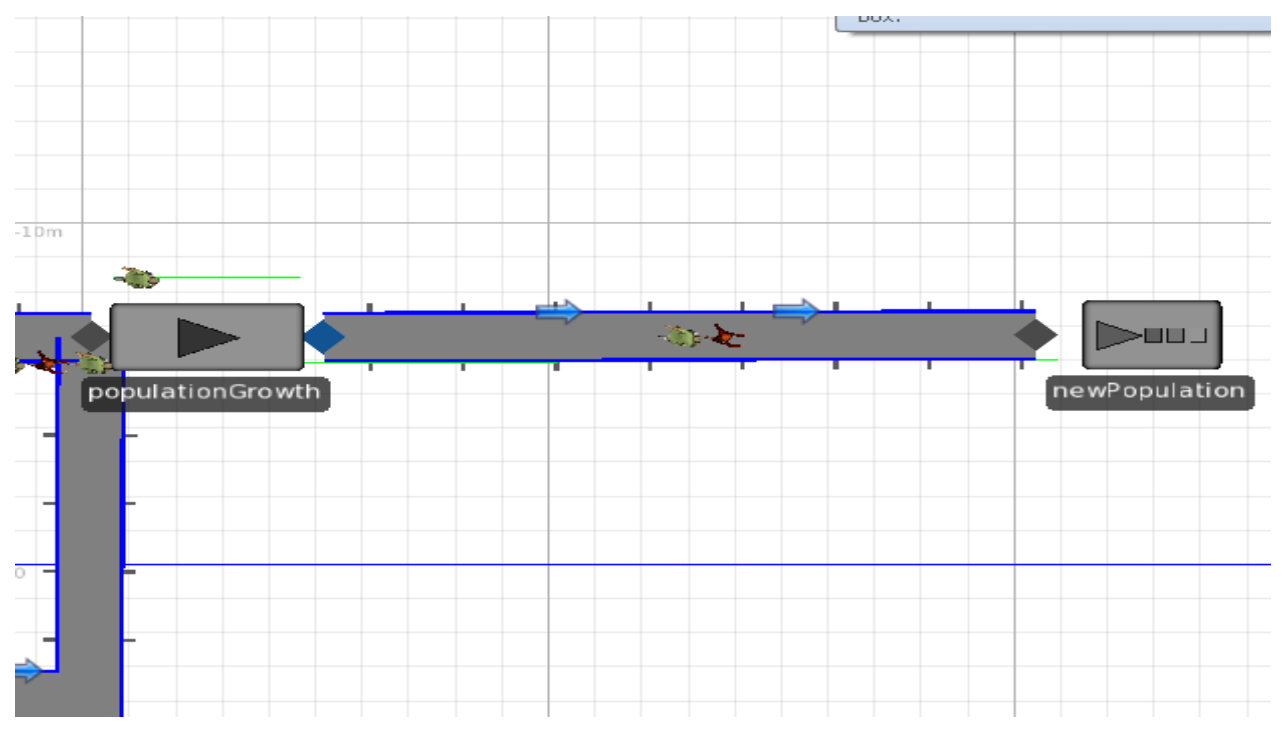

Figure 8. 

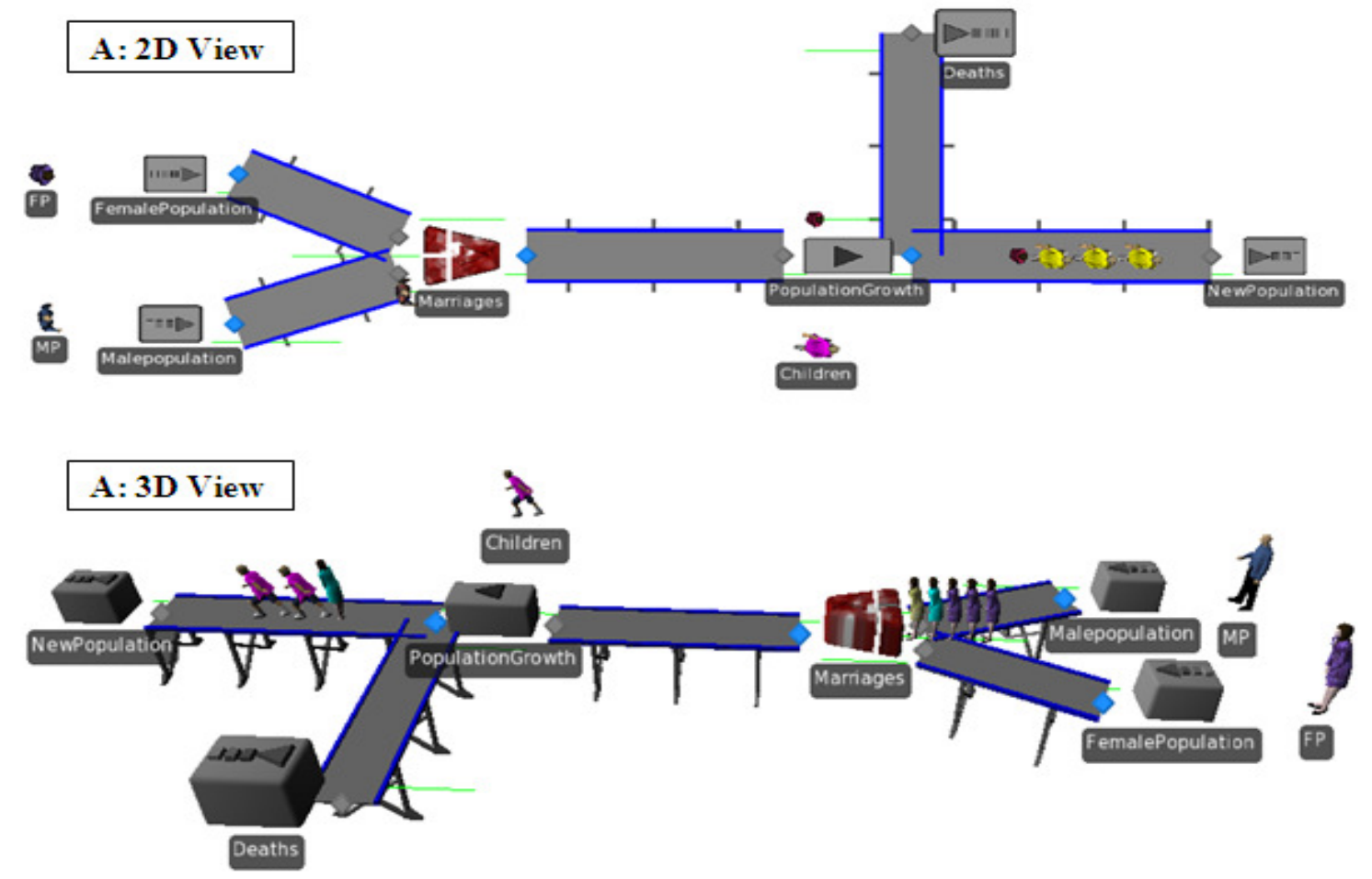

Figure 9. 


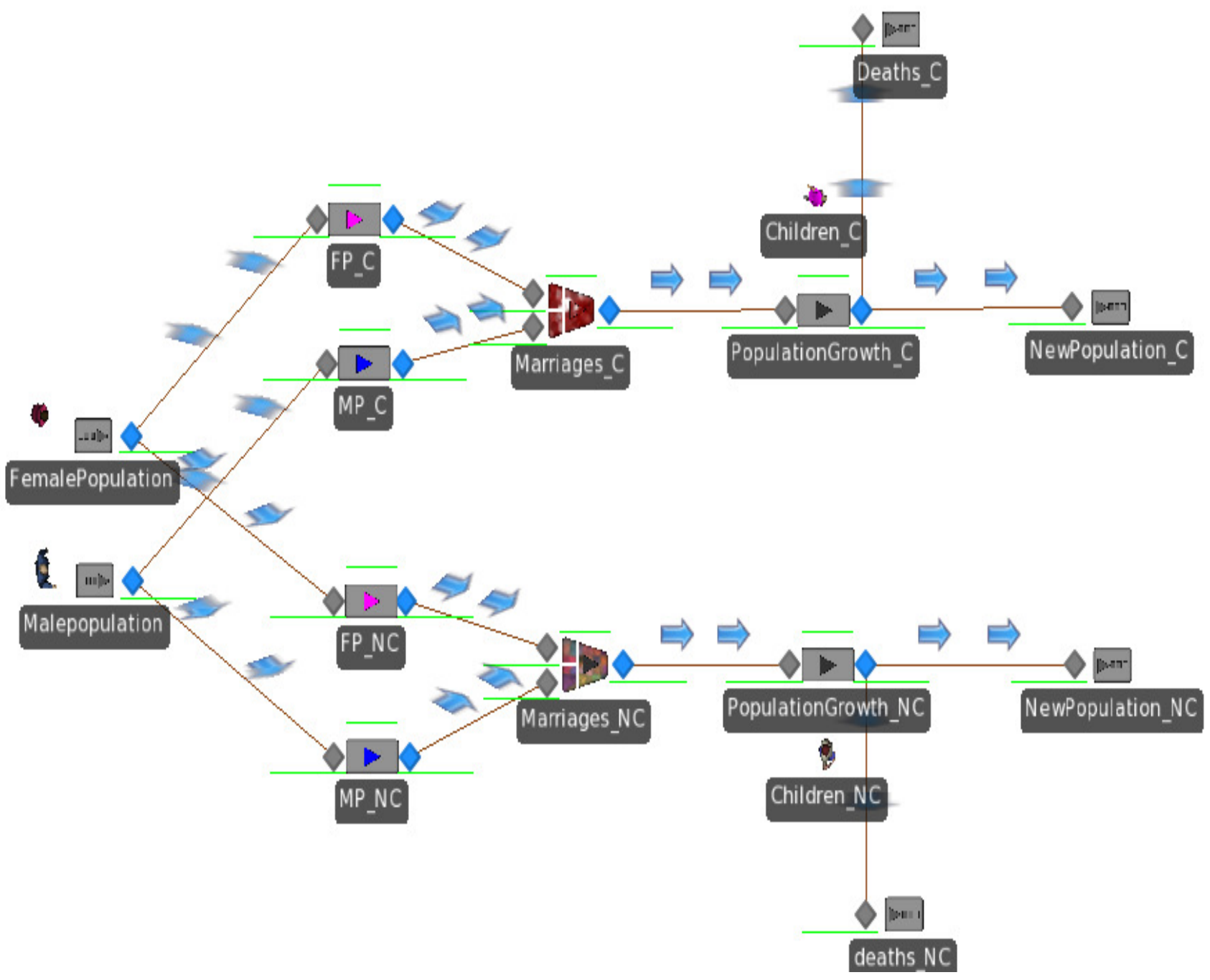

Figure 10. 


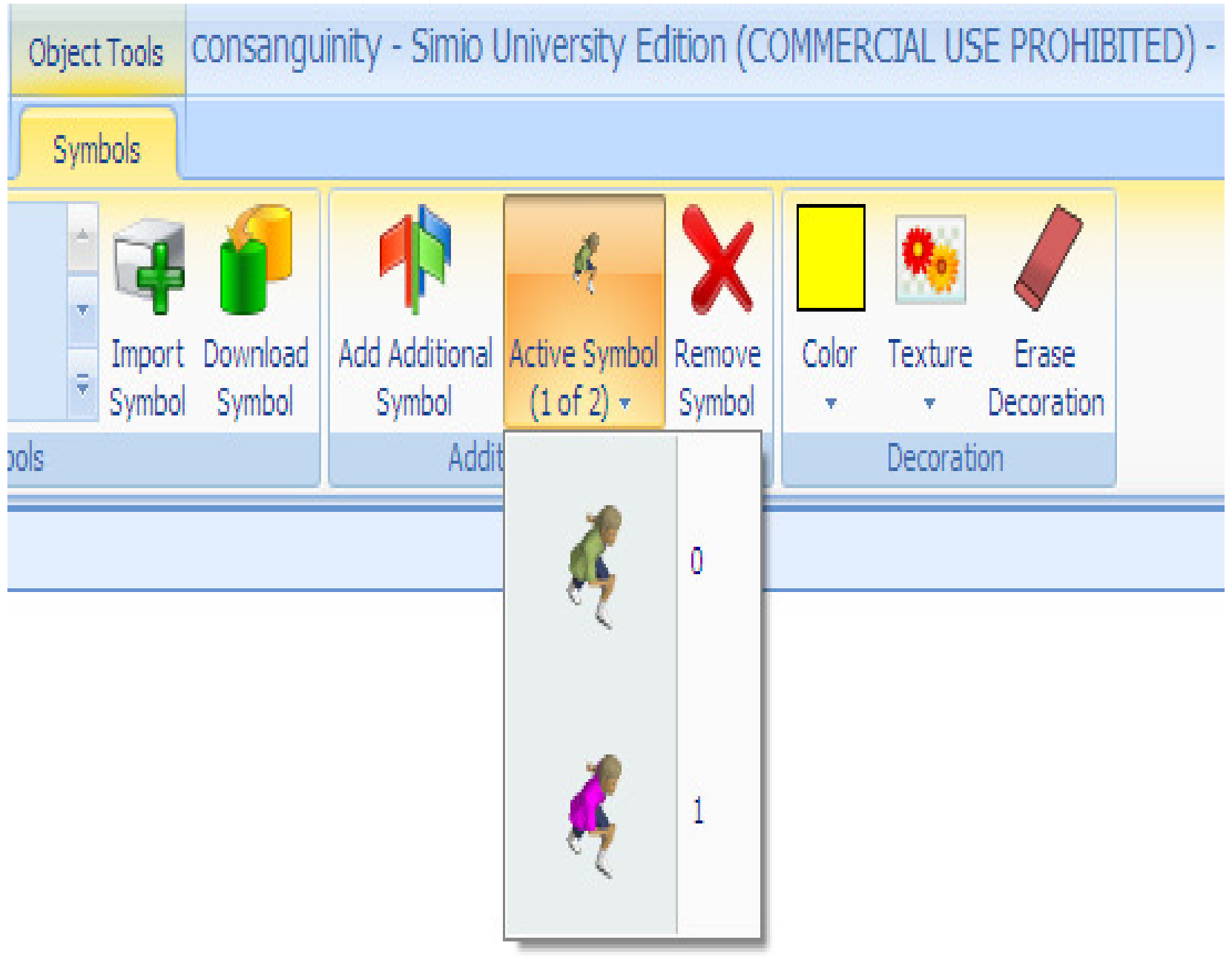

Figure 11. 


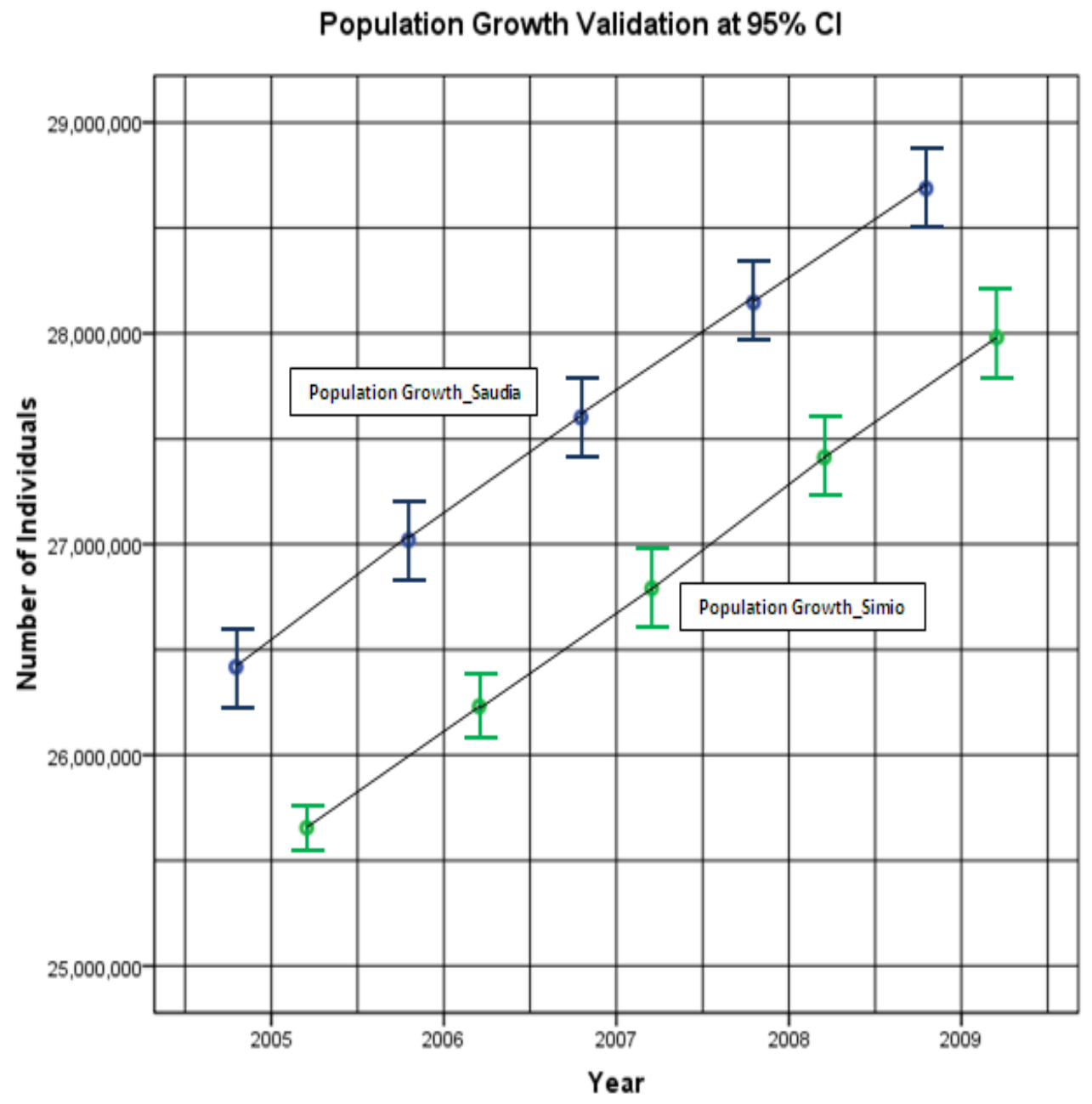

I Populationgrowth_saudia

Figure 12. 


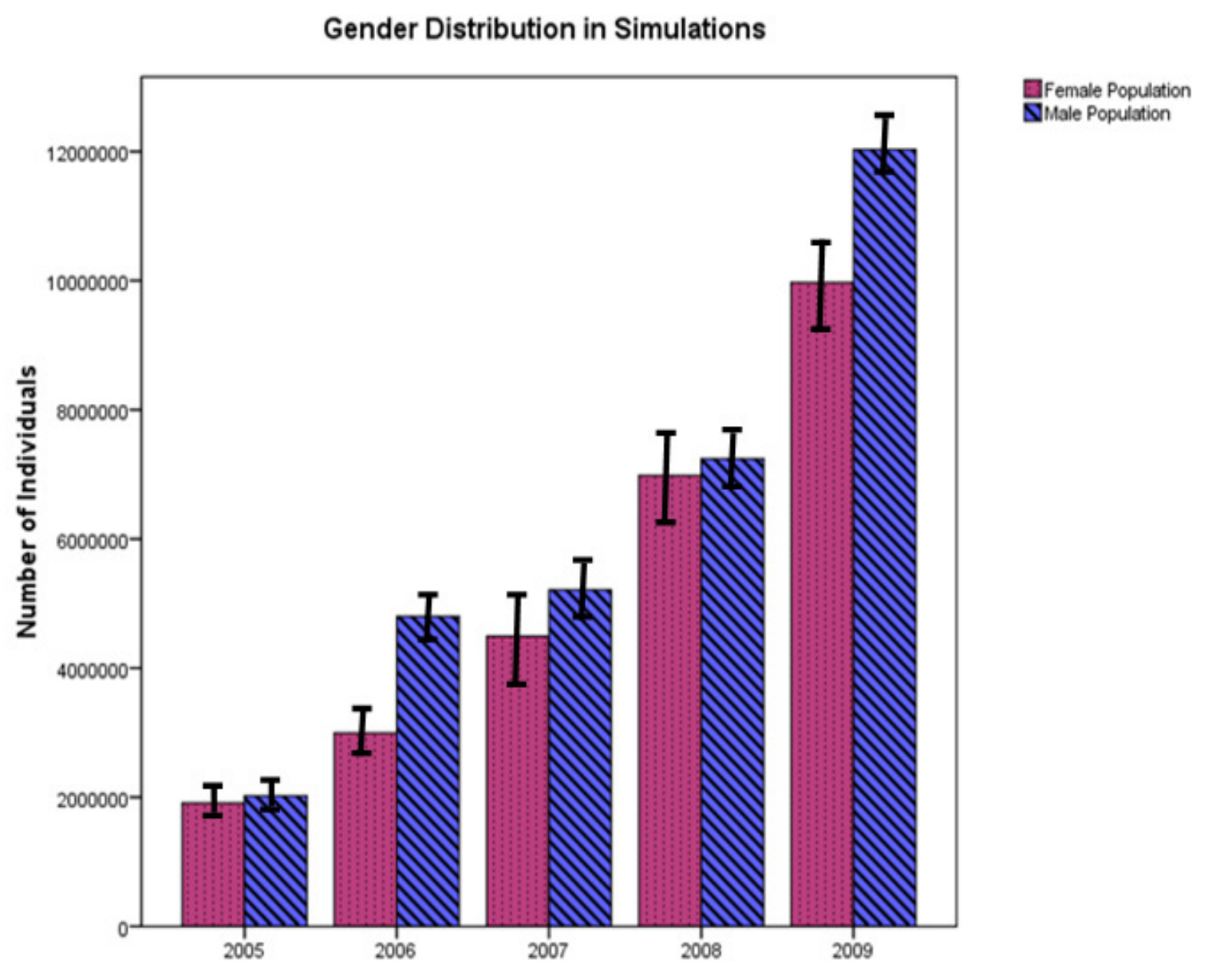

Figure 13. 


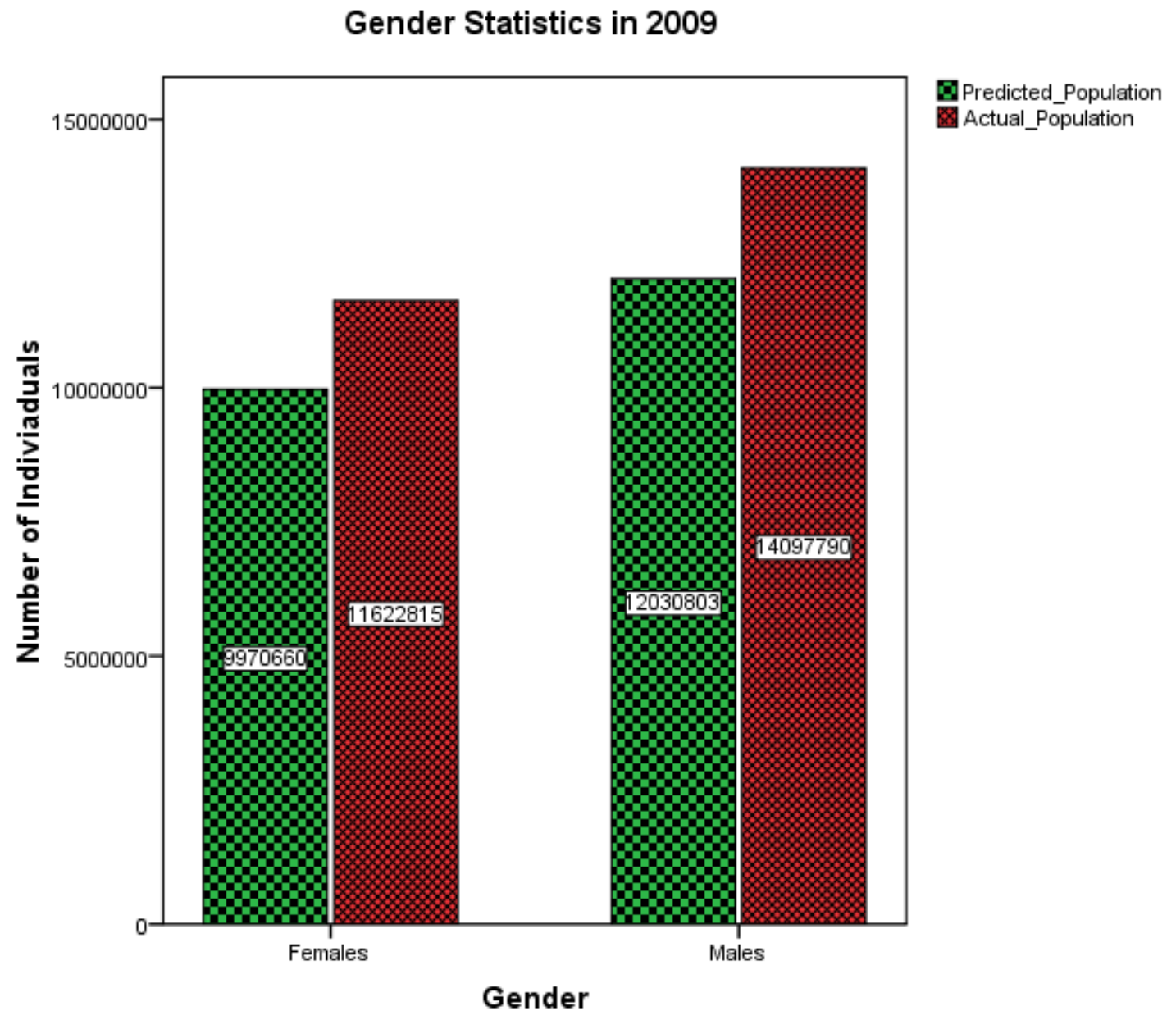

Figure 14. 